\title{
Protective Role of Bone Marrow Derived Mesenchymal Stem Cells-Conditioned Medium in the Infarcted Myocardium: The Potential Role of Selected Cytokines
}

Research Article

Amirfarhangi $\mathrm{A}^{1}$, Dezfouli $\mathrm{MG}^{2}$, Abarkar $\mathrm{M}^{3}$, Rakhshan $\mathrm{K}^{2}$, Dashti $\mathrm{S}^{2}$, Fattahi-Dolat abadi $\mathrm{M}^{4}$, Nobakht $\mathrm{M}^{5}$, Mousavi Shabestari T ${ }^{6}$, Aboutaleb N²

${ }^{1}$ Cardiology department, RasoulAkram Hospital, Iran University of Medical Sciences, Tehran, Iran.

${ }^{2}$ Physiology Research Center and Physiology Department, Faculty of Medicine, Iran University of Medical Sciences, Tehran, Iran.

${ }^{3}$ Faculty members of University of Tehran. Tehran, Iran.

${ }^{4}$ Antimicrobial Resistance Research Center, Faculty of Medicine, Iran University of Medical Sciences, Tehran, Iran.

${ }^{5}$ Department of Anatomy \&Neuroscience, Facultyof Medicine, Iran University of Medical Science, Tehran, Iran.

${ }^{6}$ Department of Immunology, Faculty of Medicine, Iran University of Medical Sciences, Tehran, Iran.

Abstract

This study examines the protective effects of mesenchymal stem cell-conditioned (CM) medium on heart tissue after induced myocardial infarction (MI). New Zealand White Rabbits were divided: MI, mesenchymal stem cells (MSC) (300 $\mu$ l), $\mathrm{CM}(300 \mu \mathrm{l})$ and Control. Echocardiography was applied, and the levels ofIL- 6 , TGF- $\beta$ and TNF- $\alpha$ were analyzed using ELISA. All tests were done 1, 4 and 8 weeks after the surgery. Histological studies were done 8 weeks after surgery. CM group showed a significant improvement in cardiac function. The level of IL-6 increased significantly in all weeks after surgery in CM. While the level of TNF- $\alpha$ increased remarkebly in CM group in $4^{\text {th }}$ week. TGF- $\beta$ decreased significantly in $\mathrm{CM}$ group at all the times. Histological findings confirmed the tissue protection effect of $\mathrm{CM}$. In conclusion, our results support the protective role of CM through its paracrine effects on the MI-induced heart.

\section{Introduction}

Myocardial infarction (MI) is the most common cause of heart failure which eventually leads to myocardial cell injury and death. It occurs as a result of cardiomyocytes death resulting in loss of viable myocytes [1]. Following MI, due to inflammatory response, the death cells are removed and replaced by fibrous scar [2]. During this process, various cytokines are produced which perform an important role in restoration of myocardial structure [3]. Moreover, since cytokines change in heart diseases, the recovery rate after treatment can be indicated by measuring the level of cytokines [4]. Cytokines are regarded as an autocrine and/or paracrine factors which can induce various biological responses. Some of heart diseases are associated with cytokine activation leading to affecting different physiologic parameters; for example cardiac mechanical function [5]. Considering the critical role of inflammation in tissue repair process, by interfering in these paths, we can decrease the heart cell injury and improve the tissue repair. Even in common treatments such as $\beta$-adrenergic blockers, the inflammatory cascades are interfered; that is, TNF- $\alpha$ and $\mathrm{TNF}-\alpha$ 1 and 2 are decreased $[6,7]$. However, several studies have shown the complicated functions of cytokine; therefore, it is difficult to anticipate the therapeutic effects of cytokines.

In spite of the fact that various kinds of treatment options have developed, the lost cardiomyocyte mass has failed to be replaced with new contractile cells. Due to the limited ability of self-regeneration of heart cells, gene-and cell-based therapeutic approaches have been introduced [8].

In the recent years, mesenchymal stem cells (MSC) have widely applied for the treatment of variety of diseases such as cancer [9], brain [10], lung [11] and liver diseases [12]. They also have generated a great amount of interests for treatment of cardiovascular disease. Due to secretion of various specific cytokines, MSCs have had different clinical applications in recent years, which one of them is their application in treatment of myocardial infarction. The goal of this method is modulating of cytokines responses

*Corresponding Author

Nahid Aboutaleb

Physiology Research Center and Physiology Department, Faculty of Medicine, Iran University of Medical Sciences, Tehran, Iran.

Tel: +989123856305

Email: dr_nabo40@yahoo.com

Received: August 29, 2016

Accepted: October 22, 2016

Published: October 26, 2016

Citation: Amirfarhangi A, Dezfouli MG, Abarkar M, Rakhshan K, Aboutaleb N, et al., (2016) Protective Role of Bone Marrow Derived Mesenchymal Stem Cells-Conditioned Medium in the Infarcted Myocardium: The Potential Role of Selected Cytokines. Int J Stem Cell Res Transplant. 4(8), 243-250. doi: http://dx.doi.org/10.19070/2328-3548-1600037

Copyright: Aboutaleb N $\mathbf{N}^{\circ}$ 2016. This is an open-access article distributed under the terms of the Creative Commons Attribution License, which permits unrestricted use, distribution and reproduction in any medium, provided the original author and source are credited. 
and as a result preventing the myocardial necrosis [13].

According to previous studies MSCs transplantation in vivo is able to provide functional cardiomyocytes as well as trigger neovascularization to provide blood vessels for nourishing the forming cardiomyocytes [14]. Although many studies on the clinical application of MSC have been carried out, the mechanisms of their functions, particularly the importance of soluble factors and their paracrine effects in MI treatment have remained elusive.

However, applications of stem cells have some disadvantages too, for example, stenting restenosis, tumorigenesis, stem cell metastasis, stem cell-mobilized cytokine-related complications [15]. Therefore, the new methods are needed to be introduced. Since stem cells usually secrete various bioactive molecules which act in paracrine fashion [16], stem cell-conditioned medium can be regarded as a good candidate for cardiac disease treatment.

The stem cell-conditioned medium therapy is much cheaper than many of other methods of treatments [17]. Moreover, it can be used several times during the treatment period, and it is more accessible [18]. Since in this method no cell is involved, the cell rejection is not expected to be seen.

In this study, we examined the possible therapeutic role of mesenchymal cell-conditioned medium during eight weeks after MI on rabbits to evaluate the effects of cytokines on the heart affected by myocardial infarction.

\section{Method and Materials}

Adult male New Zealand White rabbits with average weight of 250-300g were purchased from Pasteur Institute, Iran, and were maintained in the animal house of Medical University of Iran under constant laboratory conditions (12h light: $12 \mathrm{~h}$ dark cycle and $25-26^{\circ} \mathrm{C}$ ) with free access to food and water. All the ethics of working with laboratory animals were done in accordance with the principles of local ethical committee at the Iran University of Medical sciences. 28 male rabbits were assigned to four groups $(n=7)$ including: myocardial infarction group (MI), mesenchy-

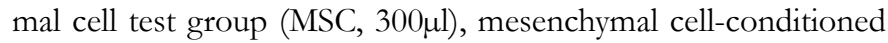
medium group (CM, $300 \mu \mathrm{l})$ and finally Normal Control group. MSCs and conditioned medium from mesenchymal stem cells were injected directly into the ventricle after inducing MI in MSC and CM groups, respectively. MI was induced according to previous studies; briefly, after anesthetizing using Ketamine $(35 \mathrm{mg} / \mathrm{kg}$ ) and Xylazine $(5 \mathrm{mg} / \mathrm{kg})$, MI was created using ligation of the left anterior descending coronary artery (LAD).

\section{Identification of MSCs}

Flow cytometry was applied for assessment of expression of superficial markers including CD105, CD166, CD90 and CD44. These markers prove the MSC identity of cells.

\section{Preparation of MSC Cell-Conditioned Medium}

The MSCs were cultured in flask and after three days the supernatant was collected and stored at $-80 \mathrm{C}$. Then, it was concentrate to the ratio of 10:1, using filter $10 \mathrm{KD}$.

\section{Echocardiography}

Heart function was measured in each group using echocardiography 1, 4 and 8 weeks after the surgery.

\section{Histological Examination}

The hearts were harvested and after tissue processing, each heart was blocked. Then $7 \mu \mathrm{m}$ thick sections were cut using a microtome. Alkaline phosphatase (ALP) was applied for determining the rate of angiogenesis in ischemic tissue; moreover, percentage of collagen fibers and pyknotic nuclei were detected using trichromemasson and Haematoxylin-Eosin (H\&E) methods, respectively. Histological studies carried out after 8 weeks after surgery.

\section{Measuring Serum Cytokines}

Several cytokines including IL-6, TGF- $\beta$ and TNF- $\alpha$ were analyzed using Enzyme-linked immunosorbent assay (ELISA). The tests were carried out based on Kit's instructions which purchased from USCN Company, China.

\section{Statistical Analysis}

One-way analysis of variance (ANOVA) and Scheffe test using the SPSS V16/0 software was used to analyze the data and $\mathrm{p}$ $<0.05$ is considered as statistically significant.

\section{Results}

\section{Histology}

Microscopic slides of heart were stained using Alkaline phosphatase which represent the level of Neo-angiogenesis in ischemic tissue (Figure 1, Graph 1), H\&E which shows the number of pyknotic cells (Figure 2, Graph 2) and trichrome masson stainingwhich indicates percentage of collagen fibers (Figure 3, Graph 3).

\section{Echocardiography}

The parameters of EF (Ejection fraction), FS (Fractional shortening) and LVED (Left Ventricle End Diastolic Dimension) were measured using echocardiography 1, 4 and 8 weeks after MI. The results are shown in the Table $1-3$.

\section{Measurement of Cytokines}

The level of IL-6, TNF- $\alpha$ and TGF- $\beta$ were measured using ELISA and results are shown in graph 4, 5 and 6, respectively.

\section{Discussion}

Our results showed that heart function improved considerably following treatment with CM. According to echocardiography results, EF in CM group improved significantly in comparison with MI and MSC groups after 4 weeks; a significant improvement was observed in FS in CM group after 1 week when compared to MI and control group. Furthermore, 4 and 8 weeks after treatment a noticeable increase in the FS parameter in CM was observed in comparison with MI and MSC groups. MSC was also able to improve the heart function; however, in most cases the CM showed 
Figure 1. Micrographs of the heart tissue $(\times 40$, Alkaline phosphatase staining) in different groups 8 weeks after inducing MI. A) Normal Control group: NO ALP reactionin the normal tissue was seen. B) MIGroup: The fibrous tissue in the heart was seen, but no ALP reaction was observed. C) MSC group: ALP reaction in capillary of heart tissue was observed. D) CM group: ALP reaction in capillary of heart tissue was less than that of in MSC group.
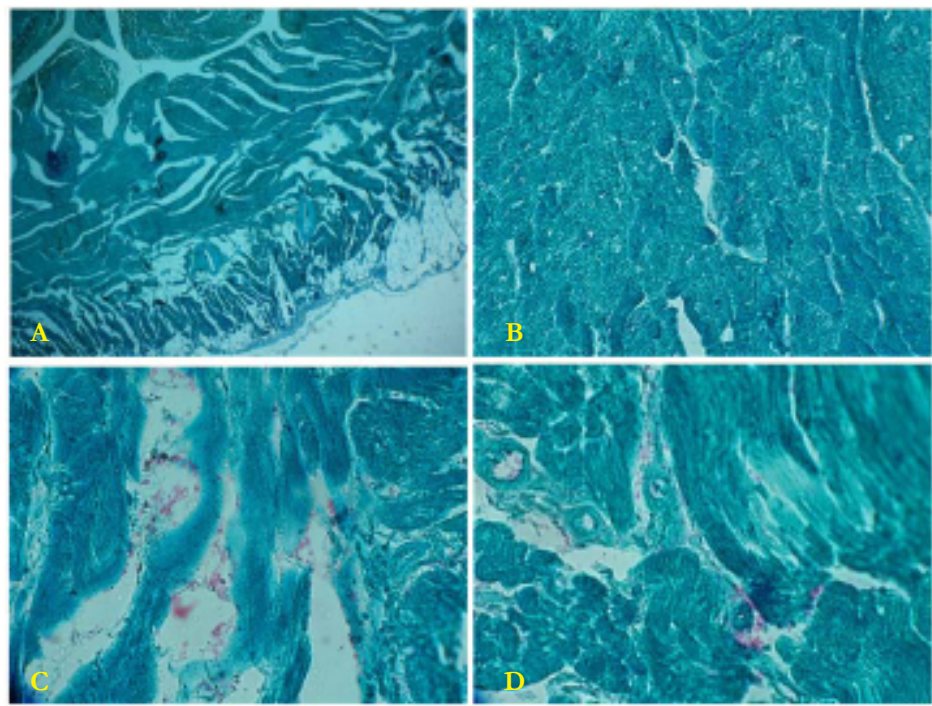

Graph 1. Alkaline phosphatase staining in heart tissue in the different groups 8 weeks after test. Percentage of staining represents the neo-angiogenesis in tissue. There was a significant difference between MSC and CM groups in comparison with MI and Control groups; moreover, a significant difference between MSC and CM was found. $* P<0.05$ vs. MI control; $* * \mathrm{P}<0.005$ vs. MI control. All groups have significant difference vs. normal control group.

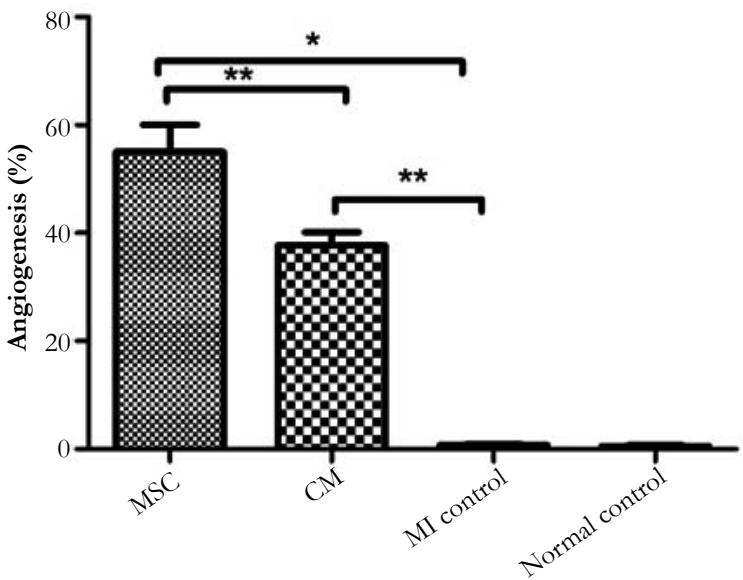

Figure 2. Micrographs of the heart tissue $(\times 40, \mathrm{H} \& \mathrm{E}$ staining) in different groups 8 weeks after test. A) Normal Control group: Presence of oval and euchromatin nuclei in heart cells representing the normal structure of heart. B) MI group: Pyknotic nucleous were seen almost in all heart cells. C) MSC group: The euchromatin and oval cell nuclei represent the repair of cardiomyocytes after treatment with MSC. D) CM group: after induction of ischemia and prescription of CM in heart cells, regeneration was seen which less than mesenchymal test group was.

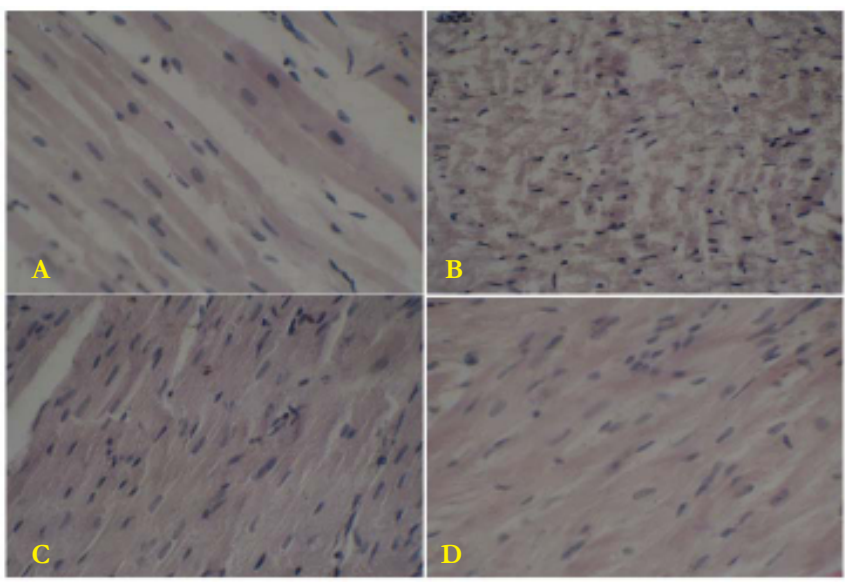


Graph 2. H\&E staining in heart tissue in the different groups 8 weeks after test. The percentage of pyknotic cells has been indicated. There was a significant difference between MSC and CM groups in comparison with MI and Control groups; moreover, a significant difference between MSC and CM was found.*P $<0.05$; $* \mathbf{P}<0.005$. All groups have significant difference vs. normal control group.

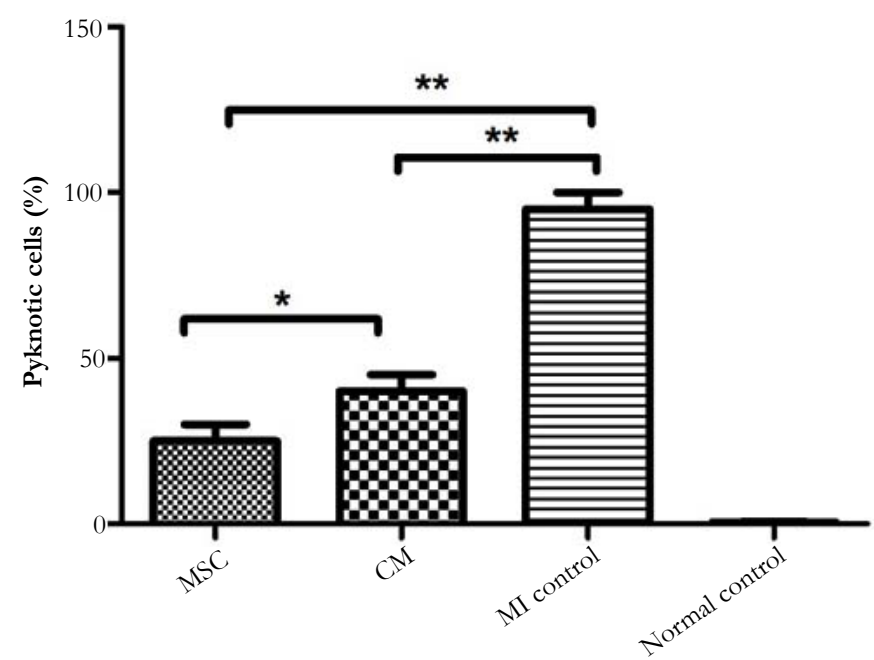

Figure 3. Micrographs of the ischemic region of heart tissue $(\times 40$, trichromemasson staining) in different groups 8 weeks after test. A) Normal Control group: representing the normal structure of heart with some collagen fibers in control group. B) MI group: fibrous tissue as well as a lot of Collagen fibers in the heart tissue was observed after induction of ischemia. C) MSC group: Representing the regeneration of Myocytes. D) CM group: Regeneration of Myocytes was seen but it was less than that of Mesenchymaltest group.

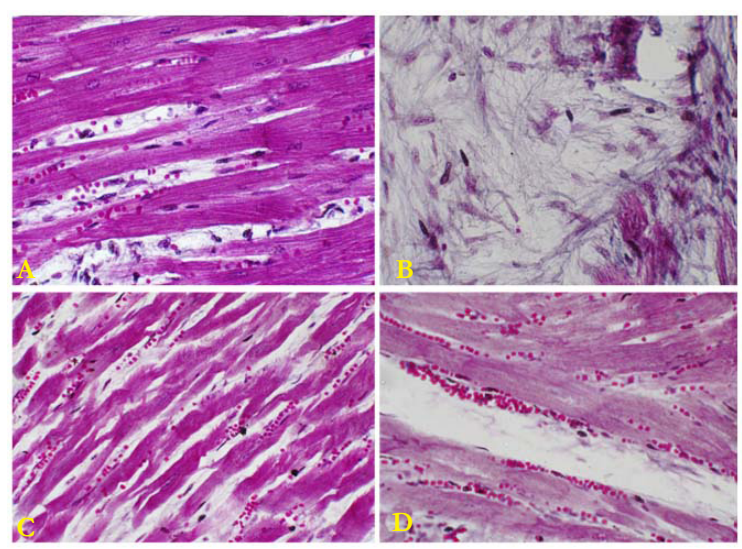

Graph 3. Massons trichrome staining in heart tissue in different groups 8 weeks after test. There was a significant difference between MSC and CM groups in comparison with MI and Control groups; moreover, a significant difference between MSC and $\mathrm{CM}$ was found. $* P<0.05 ; * * P<0.005$. All groups have significant difference vs. normal control group.

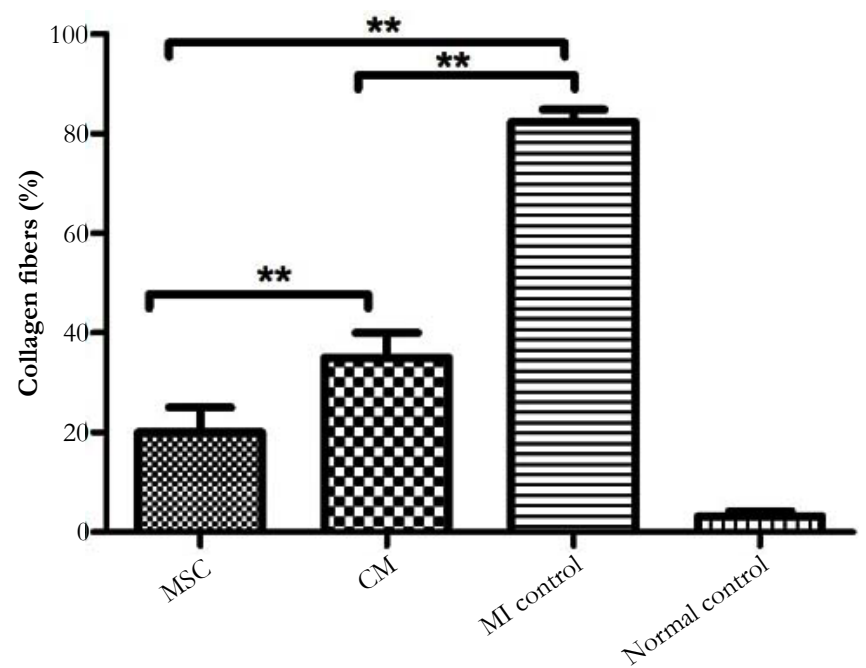


Table 1. Results in Different Groups After 1 Week.

\begin{tabular}{|c|c|c|c|}
\hline Groups & LVED $(\mathbf{m m})$ & EF $(\%)$ & FS $(\%)$ \\
\hline MSC Test & $11.73 \pm 0.33$ & $50.50 \pm 0.28$ & $23.0^{\dagger} \pm 1.21$ \\
\hline CM Test & $11.15 \pm 0.54$ & $52.25^{+\dagger} \pm 0.66$ & $29.50 \pm 0.69$ \\
\hline MI control & $12.84 \pm 1.6$ & $49.0^{+\dagger} \pm 2.31$ & $22.70^{\#} \pm 0.86$ \\
\hline Normal control & $15.0 \pm 0.69$ & $70.0 \pm 0.57$ & $40.0^{\#} \pm 0.28$ \\
\hline
\end{tabular}

Result of measurement of some parameters including: Ejection fraction (EF), Fractional Shortening (FS) and Left Ventricle End Diastolic Dimension (LVED), in various groups after 1 week after treatment. $* P<0.05$ vs. MI control; **P $<0.005$ vs. MI control; $\uparrow P<0.05$ vs. normal control; $†+P<0.005$ vs. normal control; \#P<0.05 vs. CM; \#\#P<0.005 vs. CM.

Table 2. Echocardiography Results in Different Groups After 4 Weeks.

\begin{tabular}{|c|c|c|c|}
\hline Groups & LVED & EF & FS \\
\hline MSC Test & $12.28 \pm 0.46$ & $50.83^{\#+} \pm 0.67$ & $24.0^{\#+\dagger} \pm 0.75$ \\
\hline CM Test & $12.15 \pm 1.06$ & $62.75 \pm 1.52$ & $32.50^{\dagger+} \pm 0.28$ \\
\hline MI control & $13.76 \pm 0.89$ & $46.83^{\#} \pm 0.83$ & $20.87^{\#} \pm 0.86$ \\
\hline Normal control & $14.90 \pm 1.96$ & $70.0 \pm 2.19$ & $41.20 \pm 0.57$ \\
\hline
\end{tabular}

Result of measurement of some parameters including: Ejection fraction (EF), Fractional Shortening (FS) and Left Ventricle End Diastolic Dimension (LVED), in various groups after 4 weeks after treatment. $* P<0.05$ vs. MI control; ${ }^{* *} P<0.005$ vs. MI control; $\uparrow P$ $<0.05$ vs. normal control; $+† P<0.005$ vs. normal control; $\# P<0.05$ vs. $\mathrm{CM}$; \#\#P<0.005 vs. CM.

Table 3. Echocardiography Results in Different Groups After 8 Weeks.

\begin{tabular}{|c|c|c|c|}
\hline Groups & LVED & EF & FS \\
\hline MSC Test & $13.70 \pm 0.28$ & $58.00^{\dagger} \pm 1.15$ & $29.00^{\#+\dagger} \pm 0.57$ \\
\hline CM Test & $12.50 \pm 0.86$ & $64.00 \pm 1.15$ & $33.50 \pm 0.63$ \\
\hline MI control & $13.60 \pm 0.92$ & $57.57 \pm 0.80$ & $27.14^{\#} \pm 0.55$ \\
\hline Normal control & $15.20 \pm 0.46$ & $69.50 \pm 0.80$ & $41.13^{\# \#} \pm 0.57$ \\
\hline
\end{tabular}

Result of measurement of some parameters including: Ejection fraction (EF), Fractional Shortening (FS) and Left Ventricle End Diastolic Dimension (LVED), in various groups after 8 weeks after treatment. $* P<0.05$ vs. MI control; $* * P<0.005$ vs. MI control; $\uparrow P$ $<0.05$ vs. normal control; $†+P<0.005$ vs. normal control; $\# P<0.05$ vs. $\mathrm{CM}$; \#\#P<0.005 vs. CM.

Graph 4. The level of IL-6 (pg/mg) in serum 1, 4 and 8 weeks after surgery. There was a significant difference between the CM group and other groups.

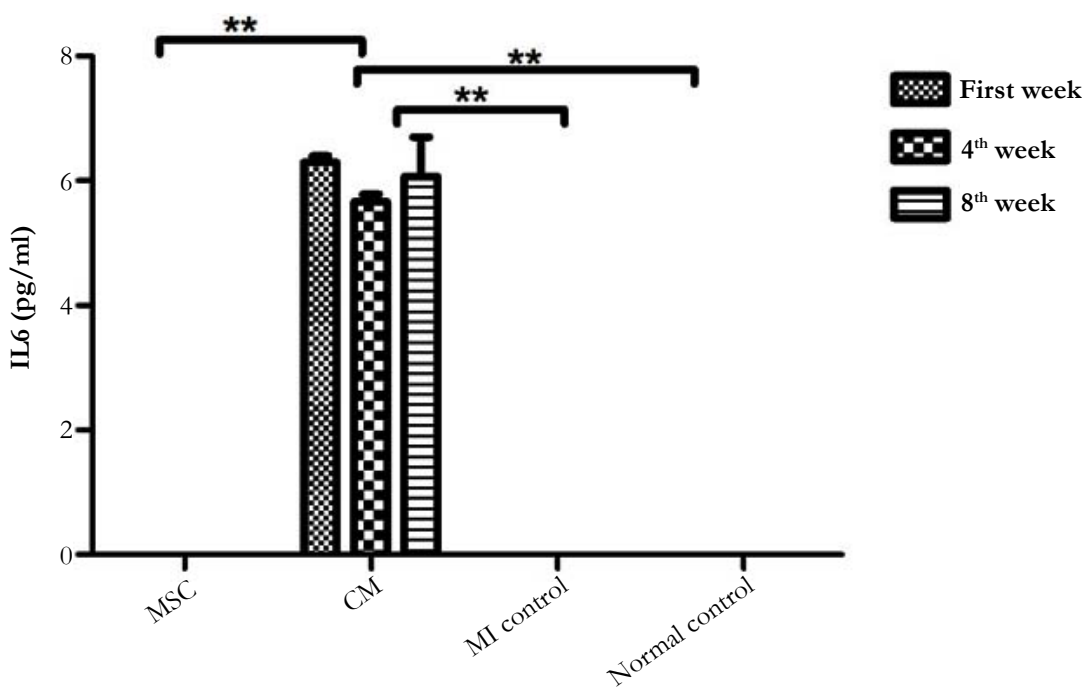


Graph 5. The level of TNF- $\alpha$ (pg/mg) in serum 1, 4 and 8 weeks after surgery. There was a significant difference between the level of TNF- $\alpha$ in MSC group in 1st and 4th weeks, and also in CM group in the 4th week, in comparison with MI and control groups. Moreover, a significant increase in the level of TNF- $\alpha$ in the MSC group in the 1th week was seen when compared to the $\mathrm{CM}$ group.

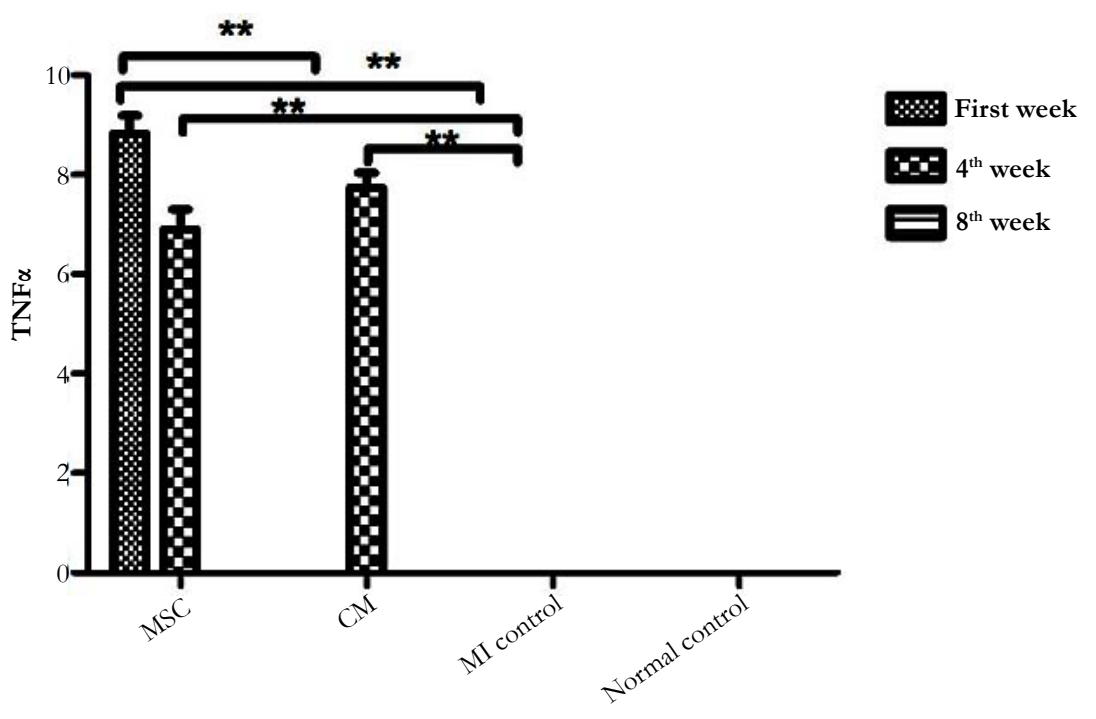

$* P<0.05 ; * * P<0.005$. All groups have significant difference vs. normal control group.

Graph 6. The level of TGF- $\beta$ (pg/mg) in serum 1,4 and 8 weeks after surgery. There was a significant difference between the levels of TGF- $\beta$ in CM group with other groups in all weeks, and also there was a significant increase in the level of TGF- $\beta$ in MSC group when compared to MI and Control groups in all weeks.
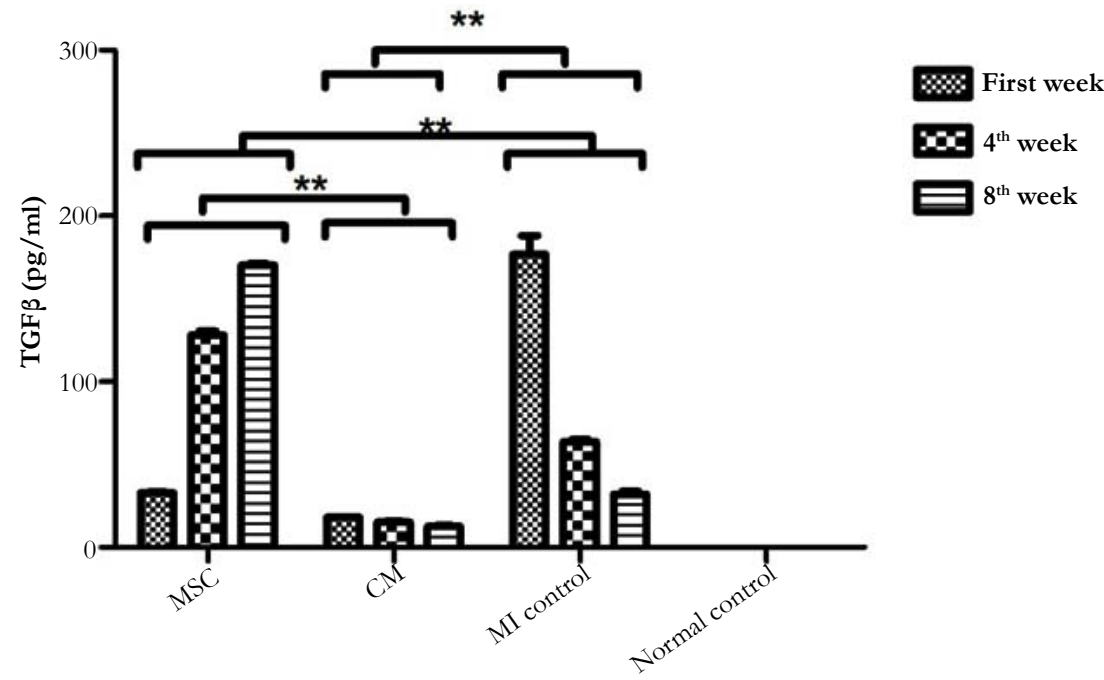

$* P<0.05 ; * * P<0.005$. All groups have significant difference vs. normal control group.

the more significant impact.

It is well documented that mesenchymal stem cells through theirparacrine action are able to preserve the function of many organs [16].

It seems specific bone marrow-derived MSC is able to secrete paracrine factors which induce angiogenesis, affect cellular migration and attenuate caspase- 3 which support the idea of cardio protective effects of MSC after MI is due to its paracrine secretions. Measurement of some factors such as VEGF, MCP-1, MIG, MIP- $1 \alpha$, and MIP- $1 \beta$ and existence of their RNA in the MSC media has been shown [19].

Timmers and et al., (2008) [23] showed that MSC-CM is able to reduce the myocardial infarct size in patients with acute MI. Moreover, the positive effects of MSC conditioned medium on ischemia-related heart dysfunction has been reported, which is in agreement with our findings. MSC-CM is shown to preserve left ventricular (LV) function in rats with induced acute myocardial infarction [20]. Cardiac fibroblasts (CFBs) have the ability to produce cytokines in response to various stimuli such as ischemia [21]. Cytokines such as MMP-2, MMP-9, and TIMP which can be secreted by MSC are able to modulate CFB activity and in this way perform an important role in regulation of left ventricular remodeling. MMP-2 after treatment with MSC-CM also prevents accumulation of collagen [22].

It is reported that MSC performs its role by secretion of paracrine mediators such as chemokines and cytokines both in the 
cultured medium and MSC-implanted area [20]. MSC can produce chemokins like SDF-1 $\alpha$, VEGF, and HGF which play an essential role in the angiogenesis, vasculogenesis and regeneration by contributing in mobilization of endothelial progenitor cell/MSC which leads to they circulate to ischemic area [19]. It is showed that MSCs through their paracrine effects lead to protection from apoptosis which results in cardiac protection [16]. Based on studies carried are so far, intracoronary treatment with MSC-CM reduces myocardial nuclear oxidative stress, expression levels of phospho-SMAD2 and active caspase 3 indicating reduction in TGF- $\beta$ signaling and apoptosis which results in considerable decrease in infarct size and improvement of systolic and diastolic cardiac performance which can be showed by echocardiography [23].

It is also demonstrated that cardiac fibrosis is considerably decreased by transplantation of MSCs. In addition, viability, alphasmooth muscle actin expression, and collagen secretion of cardiac fibroblasts are reduced by MSC-CM in vitro. It is showed that ventricular fibrosis decreased after intracardiac injection of MSCs which could lead to improvement of morphological and functional cardiac parameters [22]. Moreover, it is shown that collagen density in myocardium was lower in pigs treated with MSC-CM [24]. It is well established that MSC transplantation enhances the angiogenesis after MI [25]. The neoangiogenesis leads to reduction in apoptosis of hypertrophied myocytes in the peri-infarct region; moreover, it causes survival of viable myocardium, decrease in collagen deposition and also sustained improvement in cardiac function [26].

Capillary density enhances following treatment with human MSC conditioned medium; in addition, it maintains the systolic and diastolic function, probably by increasing myocardial perfusion, after MI in porcine model. It is suggested that mechanisms involved in vascular repair play an important role in decreasing the myocardial injury and improving cardiac function after MI [24].

In the present study the high level of IL- 6 and TNF- $\alpha$ was seen in CM group; while, the level of TGF- $\beta$ decreased significantly in this group. In MSC group, the level of TNF- $\alpha$ increased significantly in the $1^{\text {st }}$ and $4^{\text {th }}$ weeks after treatment. Moreover, the level of TGF- $\beta$ was lower than that of in MI in 1th week; however, it increased during time.

TGF- $\beta 1$ plays an important role in the development of fibrosis; therefore, it is likely that its blockage decreases or even prevents fibrosis in the infarct and the non-infarct zones and in this way leads to improvement in ventricular compliance [27].

According to previous studies, spinal cord injured rats, the level of IL-6 and TNF- $\alpha$ values show a tendency to increase after bone marrow-derived MSC-CM treatment [28]. Cytokines such as IL- 6 and TNF- $\alpha$ are mediators which play a critical role in the inflammatory response [29]. However, it is believed that the role of TNF- $\alpha$ in myocardial infarction is more complex than to be regarded just as a trigger of a cytokine cascade $[30,31]$. TNF- $\alpha$ has been found to be exist in the canine cardiac mast cells, which shows that cell-derived TNF- $\alpha$ may be released following myocardial ischemia, and therefore, indicating an 'upstream' cytokine which is responsible for initiating the inflammatory cascade [32]. It is believed that during the healing phase, TNF- $\alpha$ is expressed not only in the infarct or pre-infarct zone, but also in the normal non-infarcted myocardium, in which remodeling is occurring. Therefore, it is likely that sustained TNF- $\alpha$ expression may play a role in the repairment following myocardial infarction [33]. It is indicated that increase in the regulatory $\mathrm{T}$-cells attenuate ventricular remodeling after MI [34]. Several paracrine factors including HGF, TGF- $\beta 1$, IL- 6 , and IL-10 have been shown to be involved in the process of differentiation of T-cells into different subtypes which can be affected by MSC [35]. Monocytes which are able to differentiate into macrophage can play an important role in inflammation and tissue repair, clearing of debris and the production of pro-inflammatory cytokines, such as IL- $1 \beta$, TNF- $\alpha$, and IFN- $\gamma$. After MI, some types of macrophage have been found in the heart [36]. It is well documented that level of cytokines such as TNF- $\alpha$ or IL- 6 which are not expressed in the normal heart $[37,38]$, increase after MI which can regulate myocyte survival or apoptosis. TNF- $\alpha$ can perform its cytoprotective effect through the activation of the transcription factors $\mathrm{NF}-x \mathrm{~B}$ and stress-activated protein kinase/c-Jun N-terminal kinase (JNK) which will result in the activation of cytoprotective gene expression [39].

It is shown that TNF-R1 and TNF-R2 (TNF receptors) doublereceptor knockout mice possess larger infarct size, left coronary ligation as well as increased myocyte apoptosis in comparison with control group, which demonstrate the cardioprotective action of TNF- $\alpha$. Furthermore, it is shown that in adult feline cardiac myocyte TNF- $\alpha$ leads to increase in synthesis of structural and contractile protein $[40,41]$. These findings show that TNF- $\alpha$ may induce a cytoprotective signal which leads to preventing or delaying the development of myocyte apoptosis following MI.

It is reported that $\mathrm{TNF}-\alpha$ is able to modulate myocyte and fibroblast cell growth and function. It can stimulate proliferation and expression of fibronectin in fibroblasts. Its level has been shown to be in parallel with increasing the proliferating fibroblasts, at the border zone of the infarct region. Moreover, it is shown that TNF- $\alpha$ mRNA level is higher in comparison with control in the infarct area [33]. Since TNF- $\alpha$ induces the IL- 6 release; therefore, the increase of IL- 6 may be as result of long term presence of TNF- $\alpha[42]$.

\section{Conclusion}

As the most important finding of this study, both MSC and MSC$\mathrm{CM}$ could improve cardiac function after $\mathrm{MI}$ in rabbits, although in some cases the effect of MSC-CM was more significant. The positive effects of MSCs on the heart after MI have been demonstrated in previous studies. Furthermore, it seems MSCs can perform their role through paracrine effect. Nevertheless, considering this fact that MSC therapy may have some disadvantages. Moreover, it is likely that we can achieve better results by re-injection of MSC-CM for several times during the treatment period, while this may be risky regarding MSC therapy. Taking the cited points into account, the application of MSC-CM can be a promising approach for improvement of infarcted heart.

\section{References}

[1]. Gerdes AM, Iervasi G (2010) Thyroid replacement therapy and heart failure. Circulation. 122(4): 385-93.

[2]. Morris MW, Jr, Liechty KW (2013) Cardiac Progenitor Cells in Myocardial Infarction Wound Healing: A Critical Review. Adv Wound Care (New Ro- 
chelle). 2(6): 317-26.

[3]. Frangogiannis NG (2012) Regulation of the inflammatory response in cardiac repair. Circulation Res. 110(1): 159-73.

[4]. Martins TB, Anderson JL, Muhlestein JB, Horne BD, Carlquist JF, et al., (2006) Risk factor analysis of plasma cytokines in patients with coronary artery disease by a multiplexed fluorescent immunoassay. Am J clin pathol. 125(6): 906-13.

[5]. Bozkurt B (2000) Activation of cytokines as a mechanism of disease progression in heart failure. Ann rheum dis. 59( 1): i90-i93.

[6]. Frangogiannis NG (2008) The immune system and cardiac repair. Pharmacol Res. 58(2): 88-111

[7]. Deten A, Volz HC, Holzl A, Briest W, Zimmer HG (2003) Effect of propranolol on cardiac cytokine expression after myocardial infarction in rats. Mol Cell Biochem. 251(1-2): 127-37.

[8]. Elnakish MT, Hassan F, Dakhlallah D, Marsh CB, Alhaider IA, et al., (2012) Mesenchymal stem cells for cardiac regeneration: translation to bedside reality. Stem cells int. 2012: 646038.

[9]. Corsten MF, Shah K (2008) Therapeutic stem-cells for cancer treatment: hopes and hurdles in tactical warfare. Lancet Oncol. 9(4): 376-84.

[10]. Lindvall O, Kokaia Z (2005) Stem cell therapy for human brain disorders. Kidney Int. 68(5): 1937-1939.

[11]. Stripp BR, Shapiro SD (2006) Stem cells in lung disease, repair, and the potential for therapeutic interventions: State-of-the-art and future challenges. Am J Respir Cell Mol Biol. 34(5): 517-518.

[12]. Takami T, Terai S, Sakaida I (2012) Stem cell therapy in chronic liver disease. Curr Opin Gastroenterol . 28(3): 203-28.

[13]. Ranganath SH, Levy O, Inamdar MS, Karp JM (2012) Harnessing the mesenchymal stem cell secretome for the treatment of cardiovascular disease. Cell Stem Cell. 10(3): 244-58.

[14]. Tang YL, Zhao Q, Zhang YC, Cheng L, Liu M, et al., (2004) Autologous mesenchymal stem cell transplantation induce VEGF and neovascularization in ischemic myocardium. Regul pept. 117(1): 3-10.

[15]. Wang C-H, Cherng W-J, Verma S (2008) Drawbacks to stem cell therapy in cardiovascular diseases. Future Cardiol. 4(4): 399-408.

[16]. Tögel F, Weiss K, Yang Y, Hu Z, Zhang P, et al., (2007) Vasculotropic, paracrine actions of infused mesenchymal stem cells are important to the recovery from acute kidney injury. Am J Physiol Renal Physiol. 292(5): F1626-F1635.

[17]. Gabrielyan A, Knaak S, Gelinsky M, Arnhold S, Rösen-Wolff A (2014) Hypoxia-conditioned media allows species-specific attraction of bone marrow stromal cells without need for recombinant proteins. BMC Vet Res. 10(1): 56.

[18]. Mintz PJ, Huang K-W, Reebye V, Nteliopoulos G, Lai H-S, et al., (2014) Exploiting Human CD34+ Stem Cell-conditioned Medium for Tissue Repair. Mol Ther. 22(1): 149-59.

[19]. Boomsma RA, Geenen DL (2012) Mesenchymal stem cells secrete multiple cytokines that promote angiogenesis and have contrasting effects on chemotaxis and apoptosis. PloS one. 7(4): e35685.

[20]. Leu S, Kao Y-H, Sun C-K, Lin Y-C, Tsai T-H, et al., (2011) Myocardiumderived conditioned medium improves left ventricular function in rodent acute myocardial infarction. J Transl Med. 9(1): 11.

[21]. Porter KE, Turner NA (2009) Cardiac fibroblasts: at the heart of myocardial remodeling. Pharmacol Ther. 123(2): 255-78.

[22]. Mias C, Lairez O, Trouche E, Roncalli J, Calise D, et al., (2009) Mesenchymal stem cells promote matrix metalloproteinase secretion by cardiac fibroblasts and reduce cardiac ventricular fibrosis after myocardial infarction. Stem Cells. 27(11): 2734-2743.

[23]. Timmers L, Lim SK, Arslan F, Armstrong JS, Hoefer IE, et al., (2008) Reduction of myocardial infarct size by human mesenchymal stem cell conditioned medium. Stem cell Res. 1(2): 129-137.

[24]. Timmers L, Lim SK, Hoefer IE, Arslan F, Lai RC, et al., (2011) Human mesenchymal stem cell-conditioned medium improves cardiac function following myocardial infarction. Stem cell Res. 6(3): 206-214.

25]. Poncelet AJ, Hiel A-L, Vercruysse J, Hermans D, Zech F, et al., (2010) Intracardiac allogeneic mesenchymal stem cell transplantation elicits neoangiogenesis in a fully immunocompetent ischaemic swine model. Eur J Cardio-thoracic Surg. 38(6): 781-787.

[26]. Kocher A, Schuster M, Szabolcs M, Takuma S, Burkhoff D, et al., (2001) Neovascularization of ischemic myocardium by human bone-marrow-derived angioblasts prevents cardiomyocyte apoptosis, reduces remodeling and improves cardiac function. Nat Med. 7(4): 430-436.

[27]. Sutton MGSJ, Sharpe N (2000) Left ventricular remodeling after myocardial infarction pathophysiology and therapy. Circulation. 101(25): 29812988.

[28]. Cantinieaux D, Quertainmont R, Blacher S, Rossi L, Wanet T, et al., (2013) Conditioned medium from bone marrow-derived mesenchymal stem cells improves recovery after spinal cord injury in rats: an original strategy to avoid cell transplantation. PloS one. 8(8): e69515.

[29]. Platas J, Guillén MI, del Caz MDP, Gomar F, Mirabet V, et al., (2013) Conditioned media from adipose-tissue-derived mesenchymal stem cells downregulate degradative mediators induced by interleukin- $1 \beta$ in osteoarthritic chondrocytes. Mediators Inflamm. 2013: 357014.

[30]. Sack MN, Smith RM, Opie LH (2000) Tumor necrosis factor in myocardial hypertrophy and ischaemia - an anti-apoptotic perspective. Cardiovasc Res. 45(3): 688-695.

[31]. Belosjorow S, Schulz R, Dörge H, Schade FU, Heusch G (1999) Endotoxin and ischemic preconditioning: TNF- $\alpha$ concentration and myocardial infarct development in rabbits. Am J Physiol. 277(6): H2470-H2475.

[32]. Frangogiannis NG, Smith CW, Entman ML (2002) The inflammatory response in myocardial infarction. Cardiovasc Res. 53(1): 31-47.

[33]. Jacobs M, Staufenberger S, Gergs U, Meuter K, Brandstätter K, et al., (1999) Tumor Necrosis Factor- $\alpha$ at Acute Myocardial Infarction in Rats andÈ Effects on Cardiac Fibroblasts. J Mol Cell Cardiol. 31(11): 19491959

[34]. Matsumoto K, Ogawa M, Suzuki J-i, Hirata Y, Nagai R, et al., (2011) Regulatory $\mathrm{T}$ lymphocytes attenuate myocardial infarction-induced ventricular remodeling in mice. Int Heart J. 52(6): 382-387.

[35]. Van Den Akker F, Deddens J, Doevendans P, Sluijter J (2013) Cardiac stem cell therapy to modulate inflammation upon myocardial infarction. Biochimica Biophysica Acta. 1830(2): 2449-2458.

[36]. Lambert JM, Lopez EF, Lindsey ML (2008) Macrophage roles following myocardial infarction. Int J Cardiol. 130(2): 147-158.

[37]. Kapadia SR, Oral H, Lee J, Nakano M, Taffet GE, et al., (1997) Hemodynamic regulation of tumor necrosis factor- $\alpha$ gene and protein expression in adult feline myocardium. Circ Res. 81(2): 187-195.

[38]. Kapadia S, Lee J, Torre-Amione G, Birdsall HH, Ma TS, et al., (1995) Tumor necrosis factor-alpha gene and protein expression in adult feline myocardium after endotoxin administration. J Clin Invest. 96(2): 1042-1052.

[39]. Nian M, Lee P, Khaper N, Liu P (2004) Inflammatory cytokines and postmyocardial infarction remodeling. Circ Res. 94(12): 1543-1553.

[40]. Yokoyama T, Nakano M, Bednarczyk JL, McIntyre BW, Entman M, et al., (1997) Tumor necrosis factor- $\alpha$ provokes a hypertrophic growth response in adult cardiac myocytes. Circulation. 95(5): 1247-1252.

[41]. Kurrelmeyer KM, Michael LH, Baumgarten G, Taffet GE, Peschon JJ, et al., (2000) Endogenous tumor necrosis factor protects the adult cardiac myocyte against ischemic-induced apoptosis in a murine model of acute myocardial infarction. Proc Natl Acad Sci. 97(10): 5456-5461.

[42]. Tanabe K, Matsushima-Nishiwaki R, Yamaguchi S, Iida H, Dohi S, et al., (2010) Mechanisms of tumor necrosis factor- $\alpha$-induced interleukin- 6 synthesis in glioma cells. J Neuroinflammation. 7(1): 16. 